\title{
Orchestrating Self-Managing Systems for Autonomic Computing: The Role of Standards
}

\author{
Thomas W. Studwell \\ IBM Corporation, Autonomic Computing Architecture and Technology Group \\ 3039 Cornwallis Road, Research Triangle Park, NC 27709-2195, USA \\ studwell@us.ibm.com
}

Computing technology has progressed rapidly over the last several decades with implementations and applications that were unthinkable a decade ago now commonplace. The rate of progress, however, has brought its own cost. As large IT infrastructures grow more complex the cost of managing these systems has increased rapidly. As a result a greater percentage of the IT budget is going toward maintenance of the infrastructure rather than improving its benefit to the business.

One doesn't have to dig too deeply to understand that this increasing maintenance cost is directly related to the increase in complexity of computing technologies that have become so advanced that traditional manual management techniques are equally as apt to harm systems rather than enhance them. As each new technology strives to add enhancements for manageability, the very controls that were viewed as improving capability are actually weakening systems because the IT systems become unwieldy from the sheer number of adjustments whose interactions are unknown and virtually unknowable. Couple this increased complexity with the trend to heterogeneous distributed systems, and the complexity of the computing environments increases dramatically.

While, at first glance, the solution may seem to be a move back to very simple IT infrastructures, this is clearly not a viable alternative. The promise of e-business, distributed computing, and, eventually, Utility computing and Grid computing is just too great to ignore. For the IT Industry to bring these complex, unmanageable sytems under control it is necessary to move to self-managing systems where technology itself is used to manage technology. Autonomic systems in the human body provide the appropriate model for this progression. In this keynote, we will discuss how self-managing systems, similar to biological processes found in every living being will not only help solve today's problem of increasing complexity but, will, in fact, finally allow IT infrastructures to directly serve the corporation's business needs rather than being a static and poorly implemented attempt to automate yesterday's business processes.

This last point is vital - the IT industry must think in terms of solving business solutions rather than each supplier dwelling on the piece parts they supply to the infrastructure. This can only be accomplished through active participation in open standards where issues of interoperability and decomposition of function for self-managing systems can be properly resolved. In this keynote we will delineate the opportunities for, and challenges of, standardization of 
self-managing technologies. The IEEE will play an important role in these standardization efforts but coordination of work within the IEEE and other key computing standards bodies, such as DMTF, OASIS, GGF, and IETF, will be essential to the success of this effort. It is difficult to overstate the importance for standardization collaboration. It is not enough for localized domains to be self-managing; every component within the IT infrastructure must participate in an orchestrated way. Just as the human body has many discrete autonomic systems but all work toward a single goal, so must all the self-managing systems in the future IT infrastructure work in a uniform and predictable way.

In this keynote we will describe IBM's vision for autonomic computing and how we plan to apply the model of autonomic systems to self-managing systems with particular focus on problem determination, configuration, and optimization. At each point we will highlight the key standardization efforts underway with special emphasis on areas where standardization effort is necessary but not yet begun.

Ironically, the most prominent gap in standardization exists, not because it is perceived as unimportant, but precisely because it is recognized as essential to self-managing systems. The key area is Policy and the rules that govern selfmanaging systems. While each standards domain has a reasonable understanding of its own technology, policy related to the self-management of each domain is, to a large extent, determined by needs beyond the scope of the domain. Hence, without coordination of standards across domains the industry is deadlocked into uncertain or inadequate policy implementations. Breaking this logjam is critical to the advancement of self-managing system's standards. Further, breaking this logjam will allow the fundamental goals of the business to be converted into the standardized policies that govern the distributed IT domains throughout the enterprise.

As to the future, as self-managing systems are widely deployed, initial systems will have very rudimentary control functions. This will create new opportunities for research and product development in the area of analysis and control necessary to improve efficiency and accuracy of autonomic control. We will discuss these as well hoping to spark the interest of the DSOM community to join the call to advance the technologies of self-managing systems.

\section{Biography}

Thomas Studwell is a Senior Technical Staff Member in IBM's Autonomic Computing Architecture and Technology team. In 1975, Tom joined IBM's Thomas J. Watson Research Center and has worked for a number of IBM divisions including Research, Microelectronics, Networking Systems, and Personal Computing Devices. Tom joined IBM's Autonomic Computing Architecture and Technology group in December, 2002, and is responsible for promoting autonomic computing technologies in open standards. Tom holds several patents, is an IEEE member, and has represented IBM on IEEE, PCI SIG, and other industry consortia workgroups. 